Case

Report

\title{
Successful Rescue of a Ruptured Tracheoinnominate Fistula with Extracorporeal Membrane Oxygenation, Endovascular Stents, and Debranching Surgical Bypass
}

\author{
Yu-San Chien, MD, ${ }^{1}$ Yen-Chun Chao, MD,${ }^{2}$ Kuo-Sheng Lee, MD, ${ }^{3}$ and Kung-Hong Hsu, $\mathrm{MD}^{4}$
}

\begin{abstract}
We reported a case of ruptured tracheoinnominate fistula in a 14-year-old boy with history of repeated sternotomy. Tracheostomy was performed at age 2 years. Slide tracheoplasty was done at age 13 years. He presented to outpatient clinic with episodic hemosputum. Massive blood emanated from stoma during bronchoscopy evaluation. Venous-arterial extracorporeal membrane oxygenation was installed for resuscitation. A contrast-enhanced computed tomography (CT) and angiography confirmed the diagnosis. Immediate control of bleeding was achieved by an endovascular stent graft deployed at innominate artery. Massive hemorrhage recurred on day 7. An aortic arch stent was inserted and all arch vessels debranching via supraclavicular collar excision was performed. A covered stent was used to fenestrate the aortic stent and establish antegrade blood flow to all neck vessels via left common carotid artery. The patient remained stable at 10-month follow-up. Combination of extracorporeal membrane oxygenation, endovascular intervention, and surgical bypass could be effective in treating critical patients.
\end{abstract}

Keywords: tracheoinnominate artery fistula, tracheostomy, hemoptysis, stent graft, surgical bypass

\section{Introduction}

Tracheoinnominate artery fistula (TIF) is a rare but fatal complication of tracheostomy. The incidence ranged

${ }^{1}$ Department of Critical Care, Mackay Memorial Hospital, Taipei, Taiwan

${ }^{2}$ Department of Pediatric Cardiology, Mackay Children's Hospital, Taipei, Taiwan

${ }^{3}$ Department of Otorhinolaryngology and Head \& Neck Surgery Taipei, Taiwan

${ }^{4}$ Department of Cardiovascular Surgery, Mackay Memorial Hospital, Taipei, Taiwan

Received: January 24, 2018; Accepted: March 17, 2018

Corresponding author: Kung-Hong Hsu, MD. Department of Cardiovascular Surgery, Mackay Memorial Hospital, CVICU-B, 7F, No. 92, Sec. 2, Zhongshan N. Rd., Taipei, Taiwan

Email: erythlet@yahoo.com

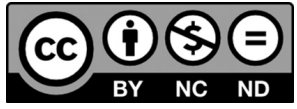

This work is licensed under a Creative Commons Attribution-NonCommercialNoDerivatives International License.

(C)2020 The Editorial Committee of Annals of Thoracic and Cardiovascular Surgery from less than $1 \%$ to $2.3 \%$ and the mortality is $100 \%$ without emergent repair. ${ }^{1,2)}$ Prompt diagnosis, effective control of bleeding, and rapid establishment of airway are critical for survival. Endovascular procedures have been reported as successful alternatives or bridges to surgery. ${ }^{3)}$ Here, we reported a case of ruptured TIF in a 14-year-old boy with long-term tracheostomy, history of repeated sternotomy, and chronic non-tuberculosis Mycobacteria tracheobronchitis. Extracorporeal membrane oxygenation (ECMO) was used for resuscitation. Then, TIF was successfully managed with emergent endovascular stent graft, followed by surgical debranching bypass and innominate artery coil embolization.

\section{Case Report}

A 14-year-old boy presented to otolaryngological clinic with episodic hemosputum for 2 days. He had history of endocardial cushion defect status post-surgical repair of atrial and ventricular septal defects and subglottic stenosis 

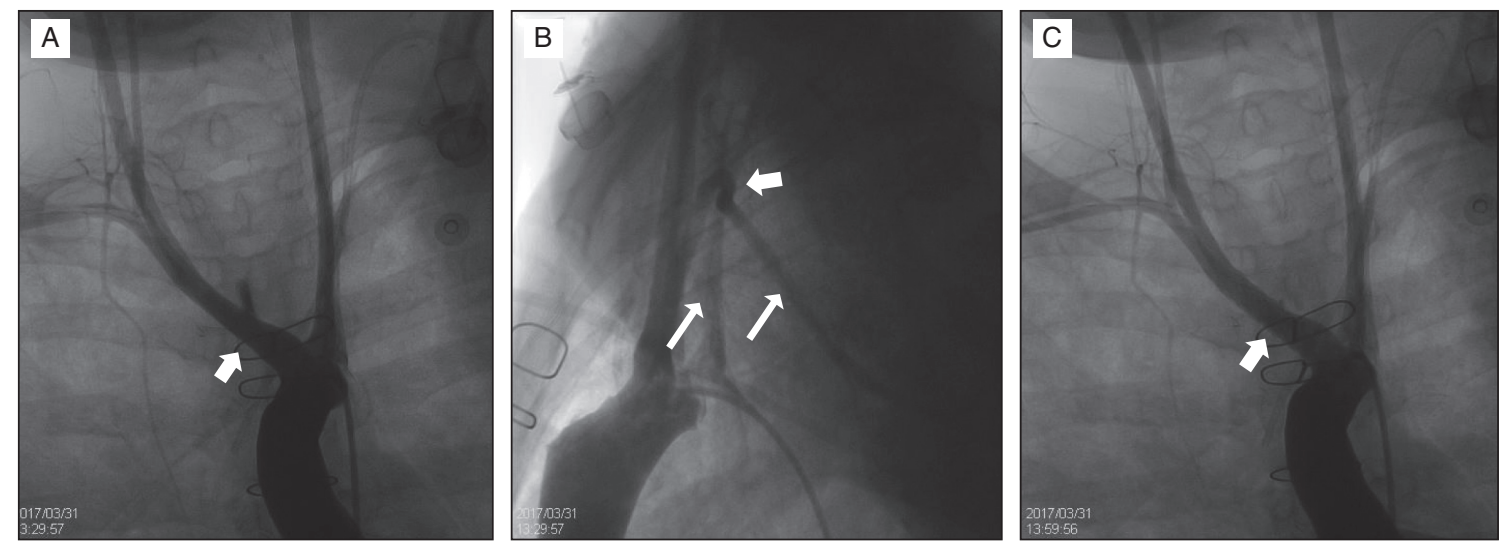

Fig. 1 Emergent angiography of ascending aorta. (A) Tracheoinnominate fistula is observed $14 \mathrm{~mm}$ distal to root of innominate artery (arrow). (B) The thick arrow indicates the fistula, and the narrow arrows indicate contrast enhancement in bronchial tree. (C) The arrow indicates a $8 / 26 \mathrm{~mm}$ balloon expandable vascular covered stent (Lifestream, Bard Ltd) deployed by the transfemoral approach. No more contrast extravasation and good flow of contrast medium through the stent graft.

status post-laryngeal reconstruction during infancy. $\mathrm{He}$ also had long-segment congenital stenosis over lower trachea and had been hospitalized 50 times due to recurrent granulation and lower airway infections. Repeated balloon dilatation and laser ablation were done but with limited effects. Tracheostomy was performed at age 2 years; T-tube or uncuffed tracheostomy tube were used with intermittent short-term positive pressure ventilator support. At age 10 years, he was diagnosed with non-tuberculosis mycobacterial tracheobronchitis; mycobacterial culture of bronchial biopsy, and tracheal aspirate taken every 3 months was persistent positive for Mycobacterium chelonae despite prolonged antibiotics treatment. Bronchoscopy showed progressive stenosis and tracheomalasia; therefore, slide tracheoplasty was done at age 13 years under cardiopulmonary bypass. The length of trachea was shortened approximately $2 \mathrm{~cm}$ after surgery.

This time at otolaryngologic clinic, massive fresh blood emanated from tracheostomy stoma during bronchoscopic evaluation. Hemostasis could not be achieved with hyperinflation of a tracheostomy tube cuff. The patient collapsed, went into pulseless electricity activity and ECMO was installed via right femoral artery and right femoral vein. A Foley catheter was inserted via tracheostomy stoma and balloon was inflated for control of bleeding. A contrast-enhanced computed tomography (CT) followed by angiography of ascending aorta confirmed the diagnosis of TIF. Emergent endovascular intervention with $8 / 26 \mathrm{~mm}$ balloon expandable vascular covered stent (Lifestream, Bard Ltd, Covington, GA, USA) was deployed to innominate artery at the cardiac catheterization lab (Fig. 1). The immediate postoperative angiography showed no more contrast enhancement in the tracheobronchial tree and the patient's hemodynamics stabilized. Because massive blood accumulated in both lungs, the patient presented with acute respiratory distress syndrome and the ratio of partial pressure of arterial oxygen and fraction of inspired oxygen $\left(\mathrm{PaO}_{2} /\right.$ $\mathrm{SpO}_{2}$ ) persistently less than $50 \mathrm{mmHg}$. Another set of venous-venous ECMO was added and $\mathrm{PaO}_{2} / \mathrm{SpO}_{2}$ improved to $80 \mathrm{mmHg}$. Bronchoscopy on hospital day 3 revealed diffuse mucosa necrosis, deformity of trachea with a $1 \mathrm{~cm}$ width defect at anterior wall at the level of tip of previous tracheostomy tube. Tracheostomy tube was placed with balloon deflated. Massive hemorrhage recurred on day 7 and an angiography revealed an active bleeder at the base of innominate artery. Surgical intervention was required. To avoid severe adhesion and fragile tissue surrounding trachea resulted from previous repeated sternotomy, an endovascular aortic arch stent was inserted and all arch vessels debranching via supraclavicular collar excision was performed under ECMO support. An $8 \mathrm{~mm}$ bypass graft (Intergard Woven, MAQUET Ltd, Rastatt, Germany) was first used to connect right subclavian, right common carotid artery, and left common carotid artery. After establishment of additional temporary cerebral perfusion via arterial cannula of venous-arterial ECMO, a thoracic aortic stent 26/21/10 (Gore TAG, Gore Ltd, Newark, NJ, USA) was place at aortic arch to exclude antegrade blood flow to TIF. Then a self-expanding covered stent (Gore Viabahn, Gore Ltd) was used to fenestrate the aortic stent and establish 


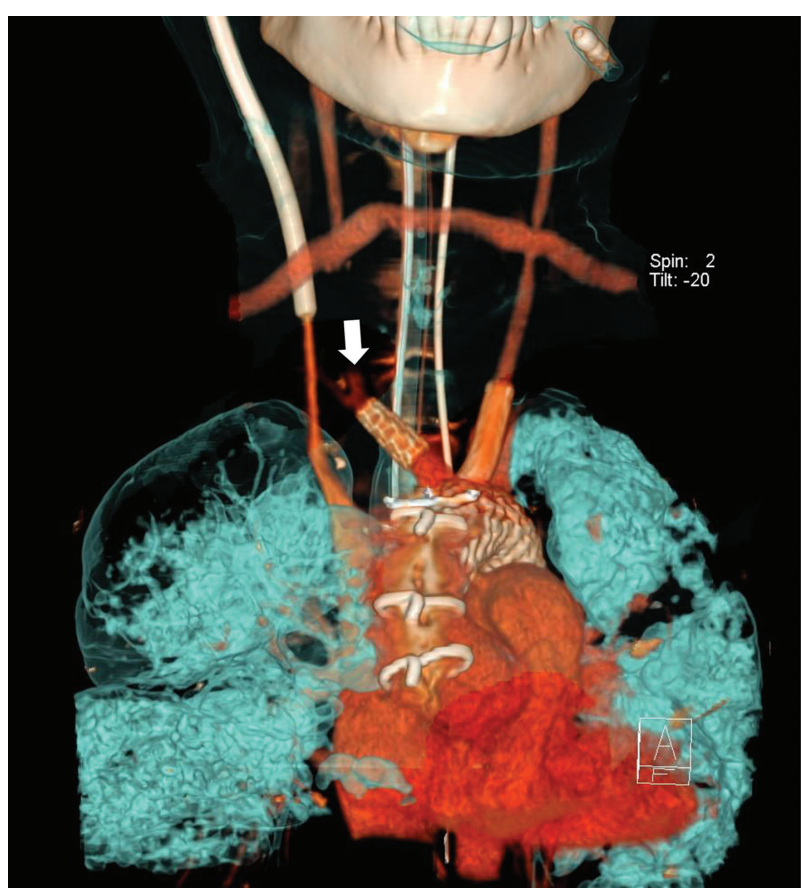

Fig. 2 Reconstructed 3D image of contrast-enhanced CT shows blood in right vertebral artery (arrow) and innominate artery. 3D: three-dimensional; CT: computed tomography

antegrade blood flow to the neck vessels via left common carotid artery. Finally, the bypass graft was anastomosed with left subclavian artery and the proximal ends of right subclavian artery and right common carotid artery were ligated to exclude retrograde flow to TIF. The defect of tracheostomy was not repaired because of severe tissue necrosis. Bleeding subsided, and the patient's oxygenation improved gradually. On day 12 , venous-venoarterial ECMO was shifted venous-venous mode. On day 17, a followed-up CT showed contrast in innominate artery, retrograde flow from right vertebral artery was suspected (Fig 2). Complete embolization of innominate artery with multiple coil 35/14/12 (Nester, Cook Medical Ltd, Bloomington, IN, USA) was done and no more contrast enhancement was found on tracheobronchial tree. On day 22, venous-venous ECMO was removed successfully. During the 22 days of ECMO use, heparin was used to maintain circuit and activated clotting time was targeted at 160-180 seconds. It was temporarily discontinued on day 7 due to recurrent bleeding and resumed after debranching bypass. Aspirin was prescribed after removal of ECMO to maintain bypass graft patent. Anti-mycobacterial antibiotics, including clarithromycin, amikacin, imipenem, and ciprofloxacin were kept for a month and shifted to a combination of clarithromycin and amikacin. He was discharged on hospital day 147 and remained stable so far. At a 10-month followup, the patient exhibited no evidence of graft infection or recurrent bleeding but had hypoxic encephalopathy with a Glasgow coma scale of $\mathrm{E} \mathrm{V}_{\mathrm{T}} \mathrm{M} 5$.

\section{Discussion}

TIF often occurred within the first 3 weeks of tracheostomy creation, ${ }^{4,5)}$ but has been reported to occur long after the procedure, especially in patients with neuromuscular disorders. ${ }^{6-8)}$ Identified risk factors included tracheostomy below the fourth tracheal ring,9) high located innominate artery, pressure necrosis of tracheal wall related to overinflated tracheostomy tube cuff, local infections, prolonged positive pressure ventilation, and spinal deformity. ${ }^{10,11)}$ Our patient developed TIF more than 12 years after tracheostomy; the slide tracheoplasty performed 2 years ago might have changed the anatomy of innominate artery relative to trachea and the chronic mycobacterial tracheobronchitis might have contributed to the gradual necrosis and structural change of trachea, eventually led to this episode of near fatal event.

Episodic hemosputum has been observed in more than $50 \%$ of patients prior to the dramatic episode. Hemorrhage with pulsation of tracheostomy tube that coincides with heartbeats (sentinel bleeding) could be observed in $35 \%$ of patients. ${ }^{12)}$ In any patient with tracheostomy and subsequent hemoptysis, TIF should have top priority on the differential.

Ideno $\mathrm{S}$. et al. successfully resuscitated two pediatric patients with TIF using ECMO. ${ }^{13)}$ They proposed that hemorrhagic shock cause by TIF is different from other etiologies because bleeding into trachea would cause ventilation and oxygenation failure, which may compromise resuscitation. In our case, with different modes of ECMO to ensure organ perfusion and oxygenation, intra-tracheal Foley catheter could be used for immediate control of bleeding despite zero pulmonary ventilation and multiple invasive procedures could be performed without concern of temporary tissue hypoperfusion.

Mainstream surgical procedures to treat TIF were ligation of innominate artery and bypass grafting of distal innominate artery ${ }^{8}{ }^{8}$ Both required median sternotomy or hemi-sternotomy and were difficult in our case because of multiple previous cardiothoracic surgeries, an endovascular stent graft implantation was performed instead. Endovascular stent graft implantation is a minimally invasive procedure that can be tolerated by critically ill patients and may be a feasible alternative to 
surgical treatment. ${ }^{3,14)}$ Complications included endograft infections and re-bleeding related to stent migration ${ }^{3)}$ or tracheal erosion. ${ }^{15)}$ Our patient had recurrent hemorrhage from innominate artery proximal to stent graft. Short landing zone of the stent along with focal tissue necrosis due to chronic and acute infection was possible contributing factors. Anticoagulant used to maintain ECMO circuit might also have increased the risk of bleeding. Surgical bypass was eventually required. If ECMO is used for resuscitation, stent graft might be better to serve as a bridge to curative surgery rather than a definitive treatment.

Transarterial embolization of the innominate artery can be used for immediate control of bleeding but it carries a high risk of cerebral ischemia or infarction. ${ }^{16)}$ In the present case, the cerebral perfusion was from left common carotid artery via the penetration stent, embolization of innominate artery helped to achieve complete hemostasis and to eliminate the possibility of rebleeding.

\section{Conclusion}

A combination of multidisciplinary treatments could be the key to successful treatment of TIF.

\section{Disclosure Statement}

None of the authors have any conflict of interest in the manuscript.

\section{References}

1) Scalise P, Prunk SR, Healy D, et al. The incidence of tracheoarterial fistula in patients with chronic tracheostomy tubes: a retrospective study of 544 patients in a long-term care facility. Chest 2005; 128: 3906-9.

2) Kurose M, Takano K, Mitsuzawa H, et al. Tracheoinnominate artery fistula with severe motor and intellectual disability: incidence and therapeutic management. Int J Pediatr Otorhinolaryngol 2014; 78: 1348-51.
3) Nakai M, Sato H, Sato M, et al. Tracheo-innominate artery fistula successfully treated by endovascular stent-graft repair. Jpn J Radiol 2013; 31: 65-70.

4) Bae MH, Lee YJ, Nam SO, et al. Endovascular stenting of tracheoinnominate fistula after tracheostomy in a 14-year-old boy. Korean J Pediatr 2016; 59: S76-9.

5) Shaukat F, Iqbal K, Akhtar S, et al. Tracheo-innominate fistula formation; a rare complication of tracheostomy. J Pak Med Assoc 2013; 63: 940-3.

6) Seung WB, Lee HY, Park YS. Successful treatment of tracheoinnominate artery fistula following tracheostomy in a patient with cerebrovascular disease. J Korean Neurosurg Soc 2012; 52: 547-50.

7) Miyake N, Ueno H, Kitano H. Pathological consideration of tracheo-innominate artery fistula with a case report. Int J Pediatr Otorhinolaryngol 2013; 77: 1322-4.

8) Furukawa K, Kamohara K, Itoh M, et al. Operative technique for tracheo-innominate artery fistula repair. J Vasc Surg 2014; 59: 1163-7.

9) McCormick B, Manara AR. Mortality from percutaneous dilatational tracheostomy. A report of three cases. Anaesthesia 2005; 60: 490-5.

10) Baydur A, Kanel G. Tracheobronchomalacia and tracheal hemorrhage in patients with Duchenne muscular dystrophy receiving long-term ventilation with uncuffed tracheostomies. Chest 2003; 123: 1307-11.

11) Ailawadi G. Technique for Managing tracheoinnominate artery fistula. Operative Techniques in Thoracic and Cardiovascular Surgery 2009; 14: 66-72.

12) Ridley RW, Zwischenberger JB. Tracheoinnominate fistula: surgical management of an iatrogenic disaster. J Laryngol Otol 2006; 120: 676-80.

13) Nakazawa K, Shinoda K. [An overview of incidence and outcome of perioperative pulmonary aspiration]. Masui 2016; 65: 4-12.

14) Deguchi J, Furuya T, Tanaka N, et al. Successful management of tracheo-innominate artery fistula with endovascular stent graft repair. J Vasc Surg 2001; 33: 1280-2.

15) Wall LP, Gasparis A, Criado E. Endovascular therapy for tracheoinnominate artery fistula: a temporizing measure. Ann Vasc Surg 2005; 19: 99-102.

16) Takasaki K, Enatsu K, Nakayama M, et al. A case with tracheo-innominate artery fistula. Successful management of endovascular embolization of innominate artery. Auris Nasus Larynx 2005; 32: 195-8. 